\title{
Caracterización de pacientes con microcarcinoma papilar de tiroides en el Hospital de Clínicas (UDELAR) en los últimos 10 años
}

Characterization of patients with papillary thyroid microcarcinoma at the Clinicas University Hospital (UDELAR)in the last 10 years

Caracterização de pacientes com microcarcinoma papilar de tireoide no Hospital de Clínicas (UDELAR) nos últimos 10 anos

Gabriela Mintegui' ${ }^{1}$, Florencia Casamayou², Claudia Arciénega², Beatriz Mendoza 3 , Alvaro Ronco ${ }^{4}$

\section{Resumen}

Introducción: el microcarcinoma papilar tiroideo (MCPT) es un tumor $\leq 1 \mathrm{~cm}$, indolente y con diagnóstico incidental o asintomático. Su incidencia aumentó últimamente por mayor detección ecográfica de nódulos no palpables, pero la mortalidad se mantiene estable, con elevada sobrevida a largo plazo.

Objetivo: caracterizar los pacientes con MCPT en la clínica de Endocrinología y Metabolismo del Hospital de Clínicas en la década 2008-2018.

Materiales y métodos: estudio observacional, transversal, retrospectivo y analítico. Se revisaron historias de archivo y policlínica de pacientes que concurrieron a nuestro servicio desde junio/2008 a junio/2018. Se recabó el número de pacientes atendidos en el hospital en 1978-2008 para estimar incidencia y casuística anual. Las asociaciones entre variables y de tendencias se analizaron estadísticamente.

Resultados: de 193 pacientes con carcinoma diferenciado de tiroides, 42 (22\%) tuvieron MCPT. La incidencia hospitalaria de MCPT tendió a aumentar en los últimos 40 años (media = 4,2 casos/año); $88 \%$ fueron mujeres. El $69 \%$ de los diagnósticos fue por punción aspiración de aguja fina (PAAF) de un nódulo tiroideo, siendo $57 \%$ unifocales y $43 \%$ multifocales. Hubo asociación significativa entre multifocalidad e invasión capsular. Un 84\% no tuvo metástasis ganglionares. En 55\% de los casos se realizó radioyodo (RY) postcirugía.

Conclusiones: aumentó la incidencia de MCPT, mayormente en mujeres. El diagnóstico prequirúrgico se hizo fundamentalmente por PAAF de nódulos tiroideos. La invasión capsular se asoció a multifocalidad. Un 16\% tuvo metástasis ganglionares al diagnóstico. En la mayoría se realizó tiroidectomía total y $55 \%$ RY.

Palabras clave: Microcarcinoma papilar de tiroides

Key words: $\quad$ Papillary thyroid microcarcinoma

\footnotetext{
1. Dra. en Medicina. Profesora Adjunta Clínica de Endocrinología y Metabolismo. Facultad de Medicina. Hospital de Clínicas (UDELAR).

2. Dra. en Medicina. Especialista de Endocrinología y Metabolismo. Facultad de Medicina. Hospital de Clínicas (UDELAR).

3. Dra. en Medicina. Profesora Clínica de Endocrinología y Metabolismo. Facultad de Medicina. Hospital de Clínicas (UDELAR).

4. Dr. en Medicina. Profesor asociado de Oncología. Facultad de Medicina CLAEH, Maldonado, Uruguay.

Los autores declaramos no presentar conflictos de interés y tampoco haber recibido apoyo o subvenciones de ningún tipo.

Correspondencia: Dra. Gabriela Mintegui. Correo electrónico: gabymin92@gmail.com

Aprobado por el Comité de Ética del Hospital de Clínicas

Recibido: 5/4/2021

Aprobado: 14/7/2021

Attribution-NonCommercial 4.0 International (CC BY-NC 4.0)
} 


\section{Introducción}

El carcinoma papilar es la neoplasia maligna más común de la tiroides. Por lo general crece lentamente y tienen muy buen pronóstico. El microcarcinoma papilar tiroideo (MCPT) se define como el carcinoma papilar de tiroides $(\mathrm{CPT})$ con diámetro $\leq 1 \mathrm{~cm}$, de acuerdo con la clasificación histológica que realizó World Health Organization $(\mathrm{WHO})^{(1)}$.

Puede ser diagnosticado en citología mediante punción aspiración por aguja fina (PAAF) guiado por ecografía, o ser un hallazgo incidental ${ }^{(2)}$. El uso extendido de la ecografía y tomografía por emisión de positrones (PET) como screening de salud ha incrementado la frecuencia de incidentalomas de tiroides, así mismo, el uso de PAAF ecoguiada ha facilitado el diagnóstico de cáncer subclínico ${ }^{(1)}$.

El presente trabajo pretende mostrar cuál es la situación de los pacientes que presentan ésta patología, cuál es su incidencia y los factores determinantes en la evolución de la enfermedad; esto permite contar con números de nuestro medio y tener conocimiento acerca de su comportamiento indolente, para así tomar algunas decisiones que plantean las guías actuales menos intervencionistas. En esta época se habla de evitar algunas tiroidectomías innecesarias y mantener un seguimiento activo para ser menos agresivos con estos tumores, dado que los tratamientos más agresivos no mejoran la sobrevida y además pueden agravar la morbilidad de los pacientes en cuestión ${ }^{(3)}$. Nuestro objetivo pretende mostrar el comportamiento indolente de este tipo de tumores, lo cual podría determinar un cambio en el tratamiento y seguimiento de esta patología. Nos planteamos como objetivos específicos los siguientes:

1. Saber si hubo aumento en el número de casos de MCPT entre 2008 y 2018.

2. Describir al diagnóstico, la forma (por punción de nódulo tiroideo o adenopatía metastásica o por hallazgo incidental) y la focalidad: uni o multifocal.

3. Determinar la distribución por edad y género y valorar cuál de los siguientes factores de riesgo presentaba cada paciente: antecedentes personales de radioterapia de cabeza-cuello, antecedentes familiares de carcinoma diferenciado de tiroides.

4. Conocer si hay asociación entre los factores que agravan el pronóstico como: multifocalidad, invasión capsular, metástasis ganglionares al diagnóstico y los factores de riesgo para CPT.

5. Establecer el tipo de tratamiento realizado: quirúrgico, radio yodo o ambos.

\section{Revisión de la literatura}

La incidencia de cáncer diferenciado de tiroides (CDT) ha aumentado de 3,6 cada 100.000 personas en 1973 a 8,7 cada 100.000 en el 2002, se considera que este incremento se debe al mayor uso de la ecografía, la cual permite la detección de nódulos no palpables. A pesar de este aumento de la incidencia, la tasa de mortalidad se ha mantenido estable, con buen pronóstico y elevada sobrevida a largo plazo.

Estudios realizados en autopsias revelan ocurrencia de MCPT de 6\% a 36\%, según el de ITO la detección en autopsias va de $0,5 \%$ a $5,2 \%{ }^{(4-7)}$.

Se ha descrito una incidencia en autopsias de $0,5 \%$ a $5,2 \%$, con multicentricidad en $15 \%$ a $44 \%$ de las lesiones y metástasis ganglionar regional en $14 \%$ a $64 \%{ }^{(2)}$. Otras series en autopsias mostraron alta prevalencia hasta $36 \%{ }^{(8)}$ y estudios de screening indican que el MCPT asintomático está presente en 5\%-10\% de la población adulta de Estados Unidos; representando cerca de 16 millones de personas con CDT no diagnosticado ${ }^{(9,10)}$.

La prevalencia de MCPT "incidental" detectado en estudio histológico de cirugías por patología tiroidea benigna es de $1,3 \%$ a $22 \%$, en comparación con la incidencia de CDT clínicamente evidente de $0,05 \%-0,1 \%$ en la población general $^{(1)}$.

Múltiples focos pueden encontrarse limitados a un lóbulo o pueden ser bilaterales. La prevalencia de multifocalidad es de $30 \%$ a $40 \%$, mientras que la enfermedad bilateral se encuentra en $20 \%$. La presencia de adenopatías metastásicas cervicales es $25 \%$ a $43 \%$ en pacientes con MCPT. La extensión extratiroidea primariamente microscópica se ha notado en $15 \%$ a $21 \%$, mientras que la invasión vascular puede estar presente en 3,5\%. Metástasis a distancia ocurre infrecuentemente, entre $1 \%$ a $2,8 \%{ }^{(8)}$.

En cuanto a su historia natural, los pacientes con MCPT tienen baja tasa de recurrencia y rara vez la enfermedad impacta en la supervivencia, con una tasa de mortalidad baja de $0,4 \%-1 \%{ }^{(8)}$. La presencia de cualquiera de los siguientes factores afecta el manejo quirúrgico del CPT y justifica la realización de una cirugía de tiroides más extensa: presencia de multifocalidad, nódulos bilaterales, metástasis cervicales, metástasis locales o a distancia, invasión local, subtipo celular agresivo de CPT (células altas, columnar, insular, pobremente diferenciado, difuso esclerosante), historia familiar de CPT y enfermedad de Graves Basedow (EGB) de base ${ }^{(8)}$.

Los factores que influyen en el riesgo de recurrencia del MCPT incluyen:

- Extensión de la cirugía: menor recurrencia si se realiza tiroidectomía total (TT) $(2,3 \%$ a $5 \%)$, frente a lobectomía e istmectomía ( $8,2 \%$ a $25 \%)$. 
- Multifocalidad: dos o más focos en el lóbulo resecado aumenta el riesgo de focos adicionales en el lóbulo contralateral y también asocia alto riesgo de invasión linfática locorregional.

- Compromiso ganglionar cervical: si existen adenopatías cervicales en la presentación del tumor, la recurrencia ganglionar a futuro es significativamente alta, al igual que si hay adenopatías subclínicas descubiertas en inspección microscópica. Si existe compromiso ganglionar cervical prequirúrgico, especialmente metástasis palpables, deben recibir disección ganglionar terapéutica.

- Metástasis a distancia: la tasa es muy baja en MCPT, $0,2 \%-2,85 \%$, pero si existen, estos tumores tienen peor pronóstico. El tratamiento debe ser agresivo incluyendo cirugía, ablación con radioyodo (RY) y otras modalidades según indicación ${ }^{(8)}$.

En el estudio de Ito, pacientes con diagnóstico de MCPT son divididos en dos grupos de opciones terapéuticas: observación y cirugía inmediata sin seguimiento previo. Se demostró que la observación puede ser una opción terapéutica para MCPT sin características desfavorables, independientemente de los antecedentes del paciente y las características clínicas. También demostró que no es demasiado tarde para realizar tratamiento quirúrgico luego que el MCPT muestre signos de progresión como aumento del tamaño tumoral y/o aparición de metástasis ganglionar durante el seguimiento $^{(11)}$.

En pacientes con MCPT con características desfavorables se recomienda tratamiento quirúrgico sin observación. Ellas son: a) localización adyacente a tráquea o superficie dorsal de un lóbulo tiroideo; b) invasión de nervio laríngeo recurrente, metástasis ganglionares en cuello; c) hallazgos citológicos que sugieran alto grado de malignidad; d) presencia de signos de progresión durante el seguimiento.

Un estudio entre 1993 y 2011 en el Hospital Kuma, muestra que en 1.235 pacientes con MCPT de bajo riesgo en observación, la proporción que progresó fue más baja en los pacientes añosos ( $\geq 60$ años) y más alta en los pacientes jóvenes ( $<40$ años); y el análisis multivariado concluyó que la edad joven es un predictor independiente de progresión de MCPT y la edad media ( $\geq 40$ años) un predictor independiente del aumento de tamaño y la progresión a enfermedad clínica ${ }^{(10,11)}$.

Los MCPT en pacientes ancianos son los más propensos a permanecer pequeños y estables, lo que sugiere que estos pacientes son los mejores candidatos para la observación; por el contrario, los MCPT de pacientes jóvenes son significativamente más agresivos, por lo que su progresión está inversamente relacionada con la edad del paciente ${ }^{(11,12)}$.

La cirugía es generalmente recomendada para CPT probado por biopsia, puede considerarse un manejo de "seguimiento activo" como alternativa a la cirugía inmediata en pacientes con tumores de muy bajo riesgo. Además, estas guías se oponen o rechazan la PAAF de nódulos tiroideos $<1 \mathrm{~cm}$ asintomáticos, incluso si existen elementos sospechosos en la ecografía, controlando con ecografías seriadas y evaluación citológica sólo si existe evidencia de progresión de la enfermedad ${ }^{(12)}$.

\section{Materiales y métodos}

Realizamos un estudio observacional, transversal, retrospectivo y analítico, aprobado por el Comité de Ética del Hospital de Clínicas. Fueron revisados cuadernos de consultas y protocolos de la Policlínica de Endocrinología y Metabolismo y libros de registro de actividad diaria del quirófano del Hospital de Clínicas desde 2008 a 2018, con datos de tiroidectomía total o parcial. Los datos entre 1978 y 2007 inclusive fueron obtenidos del Departamento de Archivo y Estadística de dicho hospital. Todos se recogieron en una tabla elaborada con ese fin.

Todo el análisis se realizó en el lenguaje de programación R (versión 3.4.1) utilizando la interfaz de Rstudio (versión 1.0.153). La asociación entre variables cualitativas se analizó con Test de Fisher incluida en la librería Stats del lenguaje R. Para el análisis de tendencia de los casos se combinó una regresión lineal y el testeo sobre los coeficientes y la simulación de 1.000 trayectorias posibles, considerando 42 casos distribuidos entre los años 2008 a 2018. Para evaluar desviaciones de lo esperado por azar se utilizaron los percentiles 2,5 y 97,5 del conteo de casos por año a partir de esas distribuciones.

\section{Resultados}

Fueron 193 pacientes con diagnóstico CPT, 151 $(78,24 \%)$ eran CPT mayor a $1 \mathrm{~cm}$ y $42(21,76 \%)$ tuvieron microcarcinoma, que fue nuestra muestra final.

Se encontró una tendencia al aumento de la incidencia de MCPT en el Hospital de Clínicas en los últimos 40 años (figuras 1 y 2). El promedio de MCPT fue de 4,2 casos/año y el $88 \%$ fueron mujeres.

Según la forma del diagnóstico se observa que el $21,4 \%(\mathrm{n}=9)$ de los casos fue de forma incidental, el $69 \%(\mathrm{n}=29)$ fue por PAAF de nódulo tiroideo y el 9,5\% $(\mathrm{n}=4)$ por ganglio metastásico. El diagnóstico por PAAF fue siempre el más frecuente, independientemente de la variante histológica (figura 3).

En cuanto la focalidad el 57,1\% fueron MCPT unifocales, mientras que el restante $42,9 \%$ multifocales, sin 


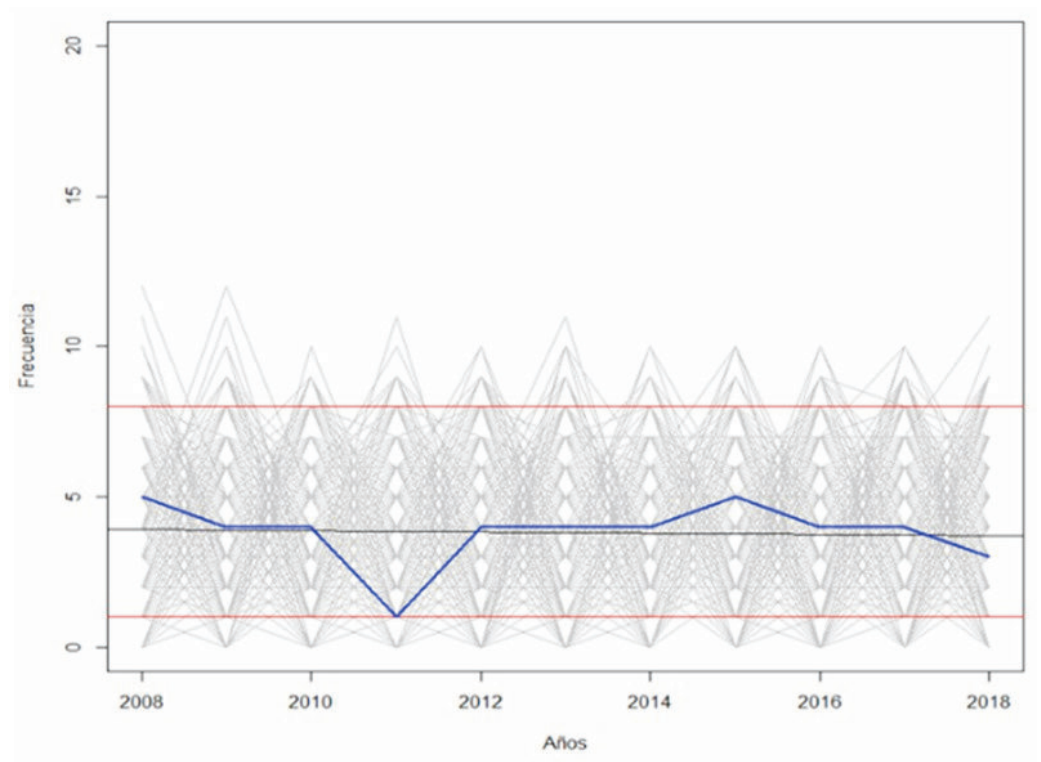

Figura 1. Frecuencia de casos en 10 años.

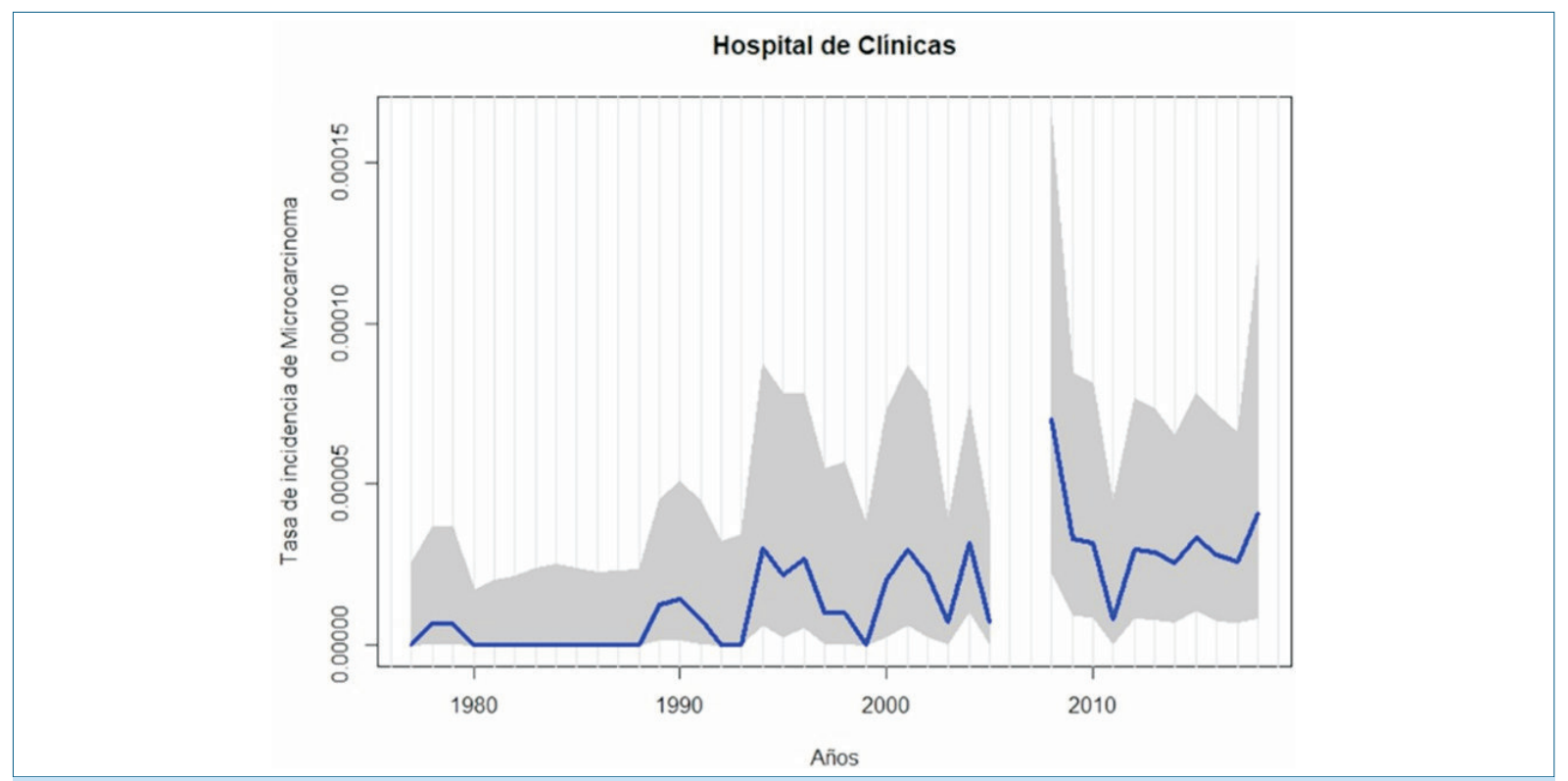

Figura 2. Tasa de incidencia de MCPT.

observarse asociación entre la forma de diagnóstico y la focalidad (p 0,70). Tampoco se observó asociación entre la forma de diagnóstico y la variante histológica ( $\mathrm{p}$ $0,14)$.

La distribución de edades no se analizó por separado para ambos sexos debido a la asimetría de los grupos (37 mujeres y 5 hombres). Sin embargo, en general, la edad al diagnóstico mostró una distribución centrada en 46,33 $\pm 13,88$ años (media \pm desvío estándar), con un rango de 22 a 69 años. Se muestra la distribución de edades en general (línea negra) y por sexo: línea roja mujeres y línea azul hombres, sin olvidar el efecto del desbalance entre hombres y mujeres (figura 4).

La distribución de pacientes con y sin registro de datos de factores de riesgo para CPT, no se diferencia entre sexos (p 1). Entre los que cuentan con dicho registro, hay diferencias entre sexos en la distribución de la presencia de factores de riesgo $(\mathrm{p}<0.05)$. Sin embargo, cabe 


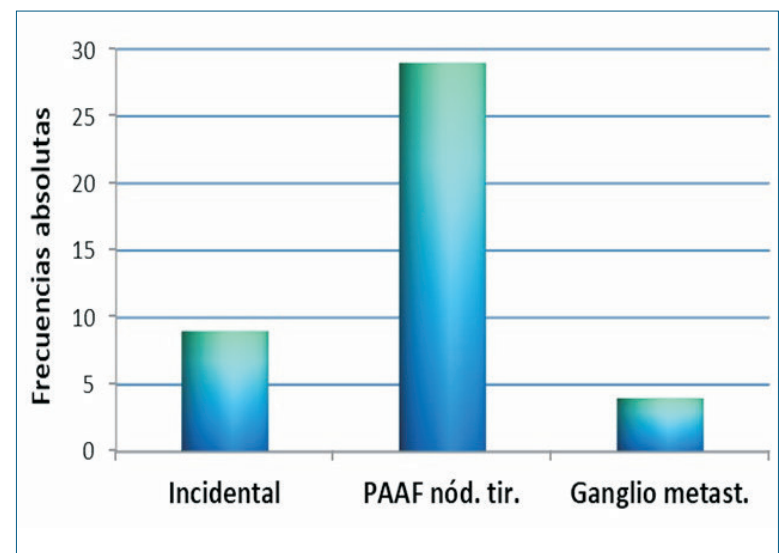

Figura 3. Formas de diagnóstico de MCPT.

Tabla 1. Factores de riesgo.

\begin{tabular}{ll}
\hline Sí & 3 \\
No & 12 \\
S/D & 27 \\
Total & 42 \\
\hline
\end{tabular}

mencionar que sólo 3 de estos pacientes sí presentaron factores de riesgo para desarrollar CPT, dos hombres y una mujer, mientras que 12 pacientes no presentaron factores de riesgo, todas mujeres (tabla 1).

De los factores que pueden agravar el pronóstico, se vio que el $42,9 \%(\mathrm{n}=18)$ presentó MCPT multifocal y $11,9 \%(\mathrm{n}=5)$ invasión capsular, observándose una asociación significativa entre la focalidad y la invasión capsular: todos los casos de invasión capsular $(\mathrm{n}=5)$ fueron en pacientes con MCPT multifocal. Sin embargo, no se observó asociación entre la focalidad y la metástasis ganglionar ( $\mathrm{p} 0,64)$.

En cuanto a la metástasis ganglionar, el 83,3\% $(n=35)$ de los pacientes no la tuvo; el 14,3\% $(n=6)$ presentó al diagnóstico y el 2,3\% $(\mathrm{n}=1)$ restante apareció en anatomía patológica luego de haberse realizado un procedimiento quirúrgico.

Cuando se correlacionó los factores de riesgo con el pronóstico, entre los pacientes con datos de factores de riesgo de CPT, ninguno presentó invasión capsular, por lo que no se encontró asociación entre la presencia de factores de riesgo de desarrollar CPT y la focalidad ( $\mathrm{p}$ 0,37 ). Sin embargo, en estos pacientes sí se observa una asociación significativa entre los factores de riesgo de CPT y la metástasis ganglionar: los tres pacientes con factores de riesgo de CPT presentaron metástasis gan-

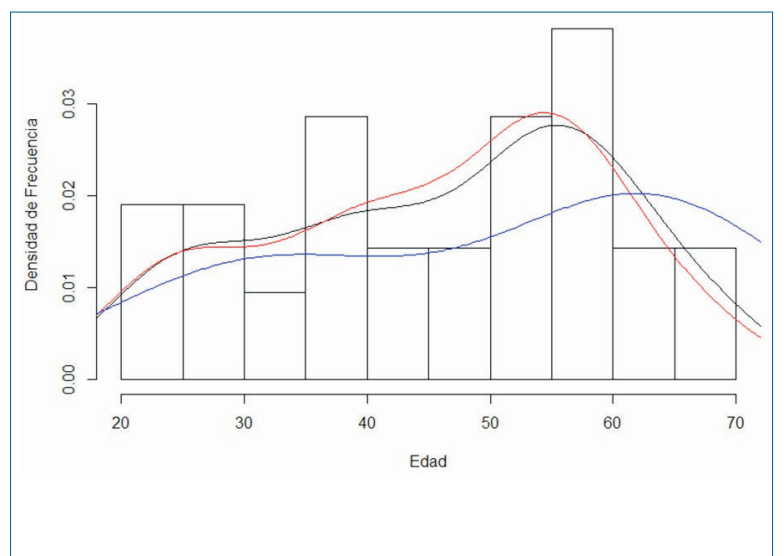

Figura 4. Frecuencia por edad.

glionar, mientras que la misma estuvo ausente en los 12 pacientes sin factores de riesgo.

En cuanto al tratamiento que recibió cada paciente, $83,3 \%(\mathrm{n}=35)$ presentó tiroidectomía total, $7,1 \%(\mathrm{n}=3)$ hemitiroidectomía y a los restantes cuatro $(9,5 \%)$ se les practicó lobectomía tiroidea.

Se vio que no hubo asociación entre el tratamiento quirúrgico realizado y la forma de diagnóstico, la variante histológica o la focalidad. En los pacientes que recibieron RY como tratamiento ablativo luego del procedimiento quirúrgico se vio que sí existe una asociación positiva con la multifocalidad. Catorce de los 23 pacientes tratados con RY presentaban MCPT multifocal, mientras que de los 19 pacientes no tratados con RY sólo 4 presentaban MCPT multifocal (figura 5).

\section{Discusión}

En trabajos internacionales, se observó un aumento de 2,4 veces entre 1973 y 2002, de 2,9 veces entre 1975 y 2009 en los Estados Unidos y de 15 veces entre 1993 y 2011 en Corea $^{(14-17)}$. En cuanto a trabajos realizados en nuestro medio, se cuenta únicamente con el trabajo de Orellano ${ }^{(18)}$, quien vio que en un periodo de 28 años desde 1977 a 2005, la prevalencia de MCPT fue de 31 casos, representando el 19,2\% del total de casos de CPT. En nuestro trabajo la cifra es mayor y se encontraron 42 casos que representan el $21,76 \%$ del total de pacientes con CPT.

En cuanto a la forma de diagnóstico, Olen y colaboradores y Carlini y colaboradores vieron que la prevalencia de MCPT diagnosticado de forma incidental luego de tiroidectomía total va desde $1,3 \%$ a $22 \%{ }^{(19-20)}$. Similar resultado se observó en nuestro estudio, 21,4\% fue diagnosticado de forma incidental y $69 \%$ fue por PAAF.

Dichos hallazgos difieren con el estudio de Orellano que mostró diagnóstico incidental en $42 \%$ y por PAAF $29 \%^{(18)}$. Creemos que la diferencia en dichos datos po- 


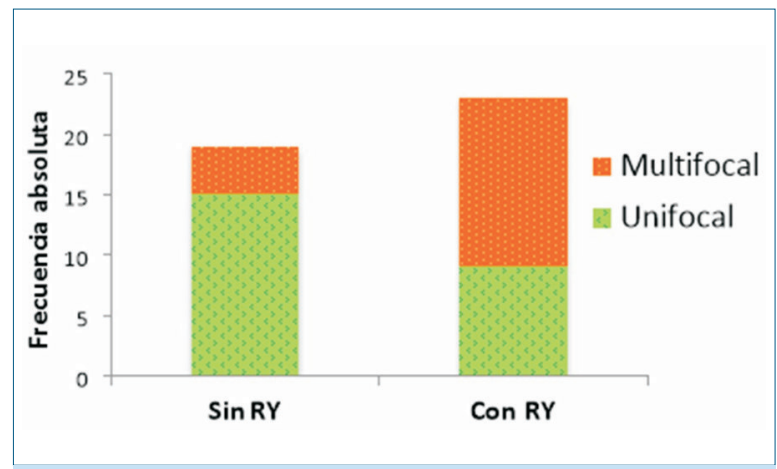

Figura 5. Relación focalidad-RY.

dría ser por un mayor uso de ecografía de cuello, con equipos de mejor resolución, así como debido al aumento de la realización de PAAF de nódulos con características ecográficas sospechas, tal como lo demostraron varios autores $^{(15,21-23)}$.

A nivel nacional los datos previos con los que contamos acerca de la focalidad de los nódulos al momento del diagnóstico corresponden a un $35 \%$ lesiones multifocales y $65 \%$ lesiones unifocales, coincidiendo con nuestro trabajo en el cual hallamos que el $42,9 \%$ son MCPT multifocales y el $57,1 \%$ son unifocales. Resultados similares se encuentran en bibliografía internacional con una prevalencia de multifocalidad de $30 \%$ a $40 \%{ }^{(18,24-26)}$.

La metástasis ganglionar cervical como primera manifestación de MCPT es extremadamente rara, sin embargo encontramos que casi el 15\% la tuvieron. Pueden ser ipsi o bilaterales y aparecen con más frecuencia en pacientes con tumor primario multifocal oculto ${ }^{(26)}$.

El CPT se presenta a una edad relativamente más joven, comparado con el resto de las enfermedades malignas. Se ha propuesto que en pacientes menores de 40 años que se presentan con una masa cervical lateral, la patología maligna tiroidea debe considerarse como primer diagnóstico. De hecho, la edad media de los casos revisados en un estudio fue menos de 40 años, mientras que MCPT sin ganglio metastásico clínicamente evidente se presenta a una edad media de 45-52 años ${ }^{(26)}$.

El carcinoma tiroideo es más frecuente en mujeres, se ha reportado una relación 3:1 en series de MCPT, representando el $70 \%-89 \%$ de los casos, dato coincidente con nuestro trabajo. En el trabajo realizado anteriormente en el Hospital de Clínicas, se hallaron resultados con predominio en el sexo femenino $90,4 \%$ frente al sexo masculino $9,6 \%{ }^{(18)}$.

La edad media al diagnóstico fue de $46,3 \pm 13,9$ años y la relación mujer:hombre de 7,4:1, coincidiendo con los datos de un estudio comparativo entre Europa y Estados Unidos que observó que la edad media al momento del diagnóstico fue de 49,4 $\pm 13,2$ años y la relación entre sexos de $5.4: 1^{(27)}$.

En nuestro estudio encontramos que $9,5 \%$ de los MCPT fueron diagnosticados por la presencia de ganglio metastásico, no contando con datos acerca de sexo, edad y focalidad en dicho grupo. Sin embargo, los casos de MCPT que se presentan con un ganglio metastásico, la predominancia de mujeres es considerablemente más baja. Esto puede estar relacionado con la agresividad de MCPT que se presenta con ganglio metastásico, así como también mayor agresividad en hombres. Datos sobre una revisión revelan varios factores de riesgo para MCPT de presentación como ganglio metastásico incluyen: edad joven, sexo masculino, multifocalidad y lesiones tiroideas primarias bilaterales ${ }^{(26)}$.

En lo relacionado a los antecedentes familiares de CPT y antecedentes de radiación de cabeza y cuello, los hallazgos en nuestro trabajo fueron que $7 \%(n=3)$ dos hombres y una mujer, presentaron dichos factores de riesgo. El 29\% (n=12) no presentan factores de riesgo. Los factores de riesgo con impacto más significativo en la sobrevida de causa específica fueron síntomas resultantes de metástasis $y / o$ invasión ${ }^{(28)}$. A diferencia de lo descrito en la literatura internacional, en nuestro estudio no se encontró asociación entre multifocalidad e invasión ganglionar linfática, hallándose sin embargo asociación significativa entre la focalidad e invasión capsular. Destacamos que solamente en $36 \%$ de los casos se pudieron recabar los datos sobre factores de riesgo, lo cual puede ser una limitante a la hora de interpretar estos datos. Hubo un trabajo que encontró sólo 1,2\% de los pacientes con enfermedad unifocal sometidos a tiroidectomía total que mostró recurrencia de la enfermedad, comparado con pacientes con enfermedad multifocal que mostraron una recurrencia de 8,6\%. Asimismo, tumores unifocales a los que se les realizó lobectomía más istmectomía mostraron una tasa de recurrencia de $3 \%$ a $4 \%{ }^{(24)}$.

Otro factor relevante es el compromiso ganglionar cervical, dado que cuando se encuentran linfadenopatías cervicales al diagnóstico del MCPT, la recurrencia ganglionar a futuro está significativamente aumentada, con una tasa de $11 \%$ - $22 \%$ en comparación a $0,8 \%$ - $6 \%$ en pacientes con ganglios negativos. Adicionalmente, los datos indican un incremento significativo en el riesgo de metástasis a distancia con la presencia de adenopatías cervicales. En comparación con el trabajo realizado previamente en población del hospital de clínicas (18) que describió invasión capsular en el 6,4\%, nuestro trabajo informa una invasión capsular del doble. La presencia de multifocalidad, edad avanzada y sexo masculino se reconocieron como factores pronósticos independientes de sobrevida libre de enfermedad; mientras que la presencia de metástasis ganglionar lateral (N1b) clínica- 
mente aparente hace más propenso a recurrencia, generalmente en ganglios linfáticos, por lo que aún con un tumor primario pequeño, debe realizarse disección linfática terapéutica en la primera cirugía en pacientes con MCPT con N1b ${ }^{(2)}$. Nuestro trabajo muestra que la población estudiada tiene una asociación significativa entre los factores de riesgo de CPT y la metástasis ganglionar, los tres pacientes con antecedentes familiares $y / 0$ antecedentes personales de irradiación de cabeza y cuello presentaron metástasis ganglionar, mientras que la misma estuvo ausente en los 12 pacientes sin factores de riesgo de CPT.

Es relevante considerar que existe relación entre la edad del paciente y la progresión del MCPT. Mazzaferri y colaboradores demostraron que la recurrencia de los CPT fue más frecuente en los extremos de la vida $(<20$ y $>59$ años), pero la tasa de mortalidad aumentó sucesivamente con la edad, a partir de los 40 años ${ }^{(29)}$. En nuestro estudio se encontraron ocho pacientes (19\%) mayores de 60 años. De estos ocho pacientes, dos fueron de hallazgo incidental, dos presentaron invasión locorregional, ninguno se presentó con ganglios metastásicos al diagnóstico, a uno se le realizó lobectomía, a siete tiroidectomía total y a dos se les realizó RY (uno de ellos con invasión locorregional). Por ende seis de ellos eran candidatos ideales para el seguimiento activo ${ }^{(8)}$.

En cuanto al tratamiento realizado en nuestra población, todos se sometieron a cirugía. Un alto porcentaje $(83,3 \%)$ se le realizó TT, $9,5 \%$ lobectomía tiroidea y $7,1 \%$ hemitiroidectomía. Dichos datos difieren de lo realizado en los años previos en el hospital de clínicas, en los cuales no se llevó a cabo ninguna lobectomía, realizándose un tratamiento más radical, la mayoría se sometieron a tiroidectomía casi total $(61,2 \%), 19,3 \%$ se realizaron tiroidectomía subtotal, e igual porcentaje tiroidectomía total ${ }^{(18)}$, que muestra la conducta menos agresiva con el correr de los años en este tipo de tumores.

Respecto a la ablación con RY postquirúrgica se utiliza para destruir cualquier remanente tiroideo luego de la TT, puede tener un efecto adyuvante en células tiroideas cancerígenas persistentes ${ }^{(30)}$. En nuestro estudio constatamos que 55\% de los MCPT recibieron RY. Notamos un cambio en la conducta terapéutica postquirúrgica con respecto a años anteriores en nuestra clínica, dado que en el trabajo de Orellano el 83,8\% de los pacientes se sometieron a dicho tratamiento ablativo, mientras que sólo $16,1 \%$ no lo hizo, concluyendo una vez más que en el pasado el tratamiento era más agresivo y radical en comparación a la actualidad.

Las guías actuales indican que pacientes con CPT no agresivo, unifocal, sin extensión locorregional o invasión, no necesitan ablación con RY, esta recomendación se basa en que no se ha encontrado mejoría en la morbilidad/recurrencia ni en la mortalidad en los pacientes con MCPT. Sin embargo, podría considerarse en pacientes con CPT con factores de mal pronóstico ${ }^{(4)}$.

Coincidiendo con lo previamente mencionado, hallamos una asociación entre RY y la presencia de multifocalidad. Se describe un $60,9 \%$ de multifocalidad en los pacientes que recibieron tratamiento ablativo.

\section{Conclusiones}

Hubo un aumento de la incidencia de MCPT. La gran mayoría fueron mujeres, con relación mujer hombre de 9:1. El diagnóstico prequirúrgico se hizo fundamentalmente por PAAF de nódulos tiroideos, seguido del hallazgo incidental. La invasión capsular se asoció a multifocalidad. Casi dos de 10 tuvieron metástasis ganglionares al diagnóstico. En la mayoría se realizó tiroidectomía total y en más de la mitad RY. No se encontró ningún caso de diseminación a distancia.

\section{Abstract}

Introduction: papillary thyroid microcarcima (PTMC) is a tumour $\leq 1 \mathrm{~cm}$ in size, indolent and with incidental or asymptomatic diagnostic. Recently, the incidence of PTMC increased as a result of higher ultrasound detection of nonpalpable nodes, although mortality rates remain stable, with high survival in the long-term.

Objective: to characterize patients with PTMC at the Endocrinology and Metabolism Department of the Clinicas University Hospital in the 2008-2018 decade.

Method: observational, transversal, retrospective and analytical study. Medical records filed and policlinic records of patients who were seen in our service since June, 2008 and June, 2018 were reviewed. The number of patients assisted in the hospital between 1978 and 2008 was found, to estimate incidence and annual casuistic. Statistical analysis of variables and tendencies was performed.

Results: 42 (22\%) out of 139 patients with poorly differentiated thyroid carcinoma (PDTC) had papillary thyroid microcarcinoma. In the last 40 years, hospital incidence of PTMC has increased (average $=4,2 \mathrm{ca}$ ses/year), $88 \%$ of which were female patients. $69 \%$ of diagnostics were done by thyroid fine-needle aspirate cytology (FNAC); 57\% were single focal and 43\% multifocal. There was a significant association between multifocality and capsule invasion. $84 \%$ of cases did not evidence lymph node metastases. In 55\% of cases postoperative radioactive iodine therapy was applied.

Conclusions: the incidence of PTMC increased, mainly in women. Diagnosis prior to surgery was mainly done by thyroid FNAC. Capsular invasion was associated to multifocality. $16 \%$ evidence lymph node 
metastases upon diagnosis. Complete thyroidectomy was done in most cases, followed by radioactive iodine therapy un $55 \%$ of cases.

\section{Resumo}

Introdução: o microcarcinoma papilar de tireoide (MCPT) é um tumor $\leq 1 \mathrm{~cm}$, indolente e com diagnóstico incidental ou assintomático. Sua incidência aumentou recentemente devido à maior detecção ultrassonográfica de nódulos não palpáveis, mas a mortalidade permanece estável, com alta sobrevida em longo prazo.

Objetivo: caracterizar os pacientes com MCPT no ambulatório de Endocrinologia e Metabolismo do Hospital de Clínicas na década de 2008-2018.

Materiais e métodos: estudo observacional, transversal, retrospectivo e analítico. Os prontuários de pacientes, históricos e atuais de pacientes foram atendidos no período junho 2008 - junho 2018 foram revisados. O número de pacientes tratados no hospital em 1978-2008 foi coletado para estimar a incidência e a casuística anual. As associações entre variáveis ??e tendências foram analisadas estatisticamente.

Resultados: de 193 pacientes com carcinoma diferenciado de tireoide, 42 (22\%) tinham MCPT. A incidência hospitalar de MCPT mostrou uma tendência crescente nos últimos 40 anos (média $=4,2$ casos/ano); $88 \%$ eram mulheres. $69 \%$ dos diagnósticos foram realizados por punção aspirativa por agulha fina (PAAF) de nódulo tireoidiano, sendo $57 \%$ unifocal e $43 \%$ multifocal. Houve uma associação significativa entre multifocalidade e invasão capsular. $84 \%$ não apresentavam metástases em linfonodos. O radioiodo pós-operatório (RY) foi realizado em $55 \%$ dos casos.

Conclusões: a incidência de MCPT aumentou, principalmente em mulheres. O diagnóstico pré-cirúrgico foi feito principalmente pela PAAF de nódulos tireoidianos. A invasão capsular foi associada à multifocalidade. 16\% tinham metástases linfonodais no momento do diagnóstico. Realizou-se tireoidectomia total na maioria dos pacientes e $55 \%$ foi submetida a RY.

\section{Bibliografía}

1. Sugitani I, Toda K, Yamada K, Yamamoto N, Ikenaga M, Fujimoto Y. Three distinctly different kinds of papillary thyroid microcarcinoma should be recognized: our treatment strategies and outcomes. World J Surg 2010; 34(6):1222-31.

2. Ito Y, Miyauchi A, Inoue H, Fukushima M, Kihara M, Higashiyama T, et al. An observational trial for papillary thyroid microcarcinoma in Japanese patients. World J Surg 2010; 34(1):28-35.

3. Pitoia F, Califano I, Vázquez A, Faure E, Gauna A, Orlandi A, et al. Consenso intersocietario sobre tratamiento y seguimiento de pacientes con cáncer diferenciado de tiroides. Rev Argent Endocrinol Metab 2014; 51(2):85-118.

4. Harach H, Franssila K, Wasenius V. Occult papillary carcinoma of the thyroid. A "normal" finding in Finland. A systematic autopsy study. Cancer 1985; 56(3):531-8.

5. Fukunaga F, Yatani R. Geographic pathology of occult thyroid carcinomas. Cancer 1975; 36(3):1095-9.

6. Samson R. Prevalence and significant of occult thyroid cancer. En: DeGroot L, ed. Radiation-associated thyroid carcinoma. New York: Grune \& Stratton, 1997:137-53.

7. Thorvaldsson S, Tulinius H, Björnsson J, Bjarnason $\mathbf{O}$. Latent thyroid carcinoma in Iceland at autopsy. Pathol Res Pract 1992; 188(6):747-50

8. Bernet $\mathbf{V}$. Approach to the patient with incidental papillary microcarcinoma. J Clin Endocrinol Metab 2010; 95(8):358692.

9. Brito J, Ito Y, Miyauchi A, Tuttle R. A clinical framework to facilitate risk stratification when considering an active surveillance alternative to immediate biopsy and surgery in papillary microcarcinoma. Thyroid 2016; 26(1):144-9.

10. Ito Y, Oda H, Miyauchi A. Insights and clinical questions about the active surveillance of low-risk papillary thyroid microcarcinomas. Endocr J 2016; 63(4):323-8.

11. Ito Y, Miyauchi A, Kihara M, Higashiyama T, Kobayashi K, Miya A. Patient age is significantly related to the progression of papillary microcarcinoma of the thyroid under observation. Thyroid 2014; 24(1):27-34.

12. Sampson R, Key C, Buncher C, Iijima S. Thyroid carcinoma in Hiroshima and Nagasaki: I. Prevalence of thyroid carcinoma at autopsy. JAMA 1969; 209(1):65-70.

13. Mehrdad K, Ghodratollah M, Tarhini M, Ali F. Papillary thyroid microcarcinoma with large and cystic neck metastasis: report of a case and review of articles. J Res Med Sci 2006; 11(5):334-8.

14. Lang W, Borrusch H, Bauer L. Occult carcinomas of the thyroid. Evaluation of 1,020 sequential autopsies. Am J Clin Pathol 1988; 90(1):72-6.

15. Davies L, Welch H. Increasing incidence of thyroid cancer in the United States, 1973-2002. JAMA 2006; 295(18):2164-7.

16. Davies L, Welch H. Current thyroid cancer trends in the United States. JAMA Otolaryngol Head Neck Surg 2014; 140(4):317-22.

17. Ahn H, Kim H, Welch H. Korea's thyroid-cancer "epidemic": screening and overdiagnosis. N Engl J Med 2014; 371(19):1765-7.

18. Orellano P, Cortez E. Microcarcinoma papilar de tiroides: caracterización clínica patológica período 1977-2005. Hospital de Clínicas. Montevideo: Facultad de Medicina, 2005. Disponible en: https://endosuem.org.uy/wp-content/uploads/ 2010/10/microcarcinoma_papilar_tiroides_ptmc.pdf. [Consulta: 7 marzo 2021].

19. Olen E, Klinck G. Hyperthyroidism and thyroid cancer. Arch Pathol 1966; 81(6):531-5. 
20. Carlini M, Giovannini C, Mercadante E, Castaldi F, Dell'Avanzato R, Zazza S. Incidental thyroid microcarcinoma in benign thyroid disease: incidence in a total of 100 consecutive thyroidectomies. Chir Ital 2006; 58(4):441-7.

21. Leenhardt $\mathbf{L}$, Grosclaude $\mathbf{P}$, Chérié-Challine L. Increased incidence of thyroid carcinoma in france: a true epidemic or thyroid nodule management effects? Report from the French Thyroid Cancer Committee. Thyroid 2004; 14(12):1056-60.

22. Burgess $\mathbf{J}$, Tucker $\mathbf{P}$. Incidence trends for papillary thyroid carcinoma and their correlation with thyroid surgery and thyroid fine-needle aspirate cytology. Thyroid 2006; 16(1):47-53.

23. Grodski S, Delbridge L. An update on papillary microcarcinoma. Curr Opin Oncol 2009; 21(1):1-4. doi: 10.1097/ CCO.0b013e32831a9a82.

24. Baudin E, Travagli J, Ropers J, Mancusi F, Bruno-Bossio G, Caillou B, et al. Microcarcinoma of the thyroid gland: the Gustave-Roussy Institute experience. Cancer 1998; 83(3):553-9.

25. Chow S, Law S, Chan J, Au S, Yau S, Lau W. Papillary microcarcinoma of the thyroid-Prognostic significance of lymph node metastasis and multifocality. Cancer 2003; 98(1):31-40.
26. Anastasilakis A, Polyzos S, Makras P, Kampas L, Valeri R, Kyriakoulis D, et al. Papillary thyroid microcarcinoma presenting as lymph node metastasis - a diagnostic challenge: case report and systematic review of literature. Hormones (Athens) 2012; 11(4):419-27.

27. Malandrino P, Pellegriti G, Attard M, Violi M, Giordano C, Sciacca L, et al. Papillary thyroid microcarcinomas: a comparative study of the characteristics and risk factors at presentation in two cancer registries. J Clin Endocrinol Metab 2013; 98(4):1427-34.

28. Roti E, degli Uberti E, Bondanelli M, Braverman L. Thyroid papillary microcarcinoma: a descriptive and meta-analysis study. Eur J Endocrinol 2008; 159(6):659-73.

29. Mazzaferri E. Management of low-risk differentiated thyroid cancer. Endocr Pract 2007; 13(5):498-512.

30. Haugen B, Alexander E, Bible K, Doherty G, Mandel S, Nikiforov Y, et al. 2015 American Thyroid Association Management Guidelines for adult patients with thyroid nodules and differentiated thyroid cancer: The American Thyroid Association Guidelines Task Force on Thyroid Nodules and Differentiated Thyroid Cancer. Thyroid 2016; 26(1):1-133. doi: $10.1089 /$ thy.2015.0020.

\section{Contribución de autores}

Gabriela Mintegui, ORCID 0000-0002-9747-176X. Concepción, diseño, análisis, interpretación de los resultados, redacción, revisión crítica. Florencia Casamayou, ORCID 0000-0002-7075-2537. Concepción, ejecución, análisis, interpretación de los resultados, redacción, revisión crítica.

Claudia Arciénega, ORCID 0000-0002-1062-156X. Concepción, ejecución, análisis, interpretación de los resultados, redacción, revisión crítica. Beatriz Mendoza, ORCID 0000-0002-7898-9336. Concepción, diseño, redacción, revisión crítica.

Álvaro Ronco, ORCID 0000-0002-6328-1482. Análisis, interpretación de los resultados, revisión crítica. 